\title{
Riesgo de violencia contra la pareja en mujeres gestantes y lactantes en Sincelejo, Colombia
}

\author{
Trabajo Resultados de Investigación \\ Liliana Margarita Meza-Cueto ${ }^{3}$, Luz Marina Orrego-Medina ${ }^{4}$, Keyla \\ Coronado-Almendrales ${ }^{5}$
}

\section{Introducción}

Para profundizar en el tema de la violencia contra la mujer, es necesario el conocimiento previo de su definición: "El concepto de violencia, ha ido evolucionando con el tiempo" (Cirici , Querol, y Ripoll, 2010, p. 550). Hoy en día, en Colombia se define el término violencia contra la mujer como "cualquier acción u omisión que le cause muerte, daño o sufrimiento físico, sexual, psicológico, económico o patrimonial por su condición de mujer, así como las amenazas de tales actos, la coacción o la privación arbitraria de la libertad, bien sea que se presente en el ámbito público o en el privado" (Ley No 1257, 2008, párr. II).

La problemática de la violencia que existe en contra de la mujer, es un tema que ha adquirido una alta relevancia en la sociedad; genera impacto en la gestión, intervención y control por parte del sistema de salud y entes reguladores que buscan mini-

${ }^{3}$ Magister en Psicología, Universidad del Norte. Psicóloga. Docente investigadora de la Corporación Universitaria del Caribe-CECAR, Sincelejo, Colombia. Liliana.mezac@cecar.edu.co

4 Magister en Psicología clínica, Universidad del Norte, Colombia. Psicóloga. Docente de la Universidad Nacional Abierta y a Distancia-UNAD, Corozal, Colombia. Luz.orrego@unad.edu.co

5 Especialista en gerencia de la calidad y auditoria en salud, Universidad del Norte, Colombia. Psicóloga. Docente de la Universidad Nacional Abierta y Distancia-UNAD, Corozal, Colombia. Keyla.coronado@unad.edu.co 
mizar el riesgo de ocurrencia de este acto de violencia. Es válido precisar que el estado y los entes que vigilan la violencia contra la mujer, han implementado medidas o mecanismos para disminuir las cifras de violencia, pero estos mecanismos existentes siguen siendo altamente cuestionables debido a las estadísticas y cifras alarmantes que aún se reportan en los medios de los órganos de control. Aunque los órganos de control sí realizan esfuerzos a nivel institucional desplegándolos en el ámbito normativo y ha implementado mejoras en las políticas públicas y de gestión, aún estos métodos se encuentran disímiles y desencajados entre las distintas partes involucradas, y su impacto en la positiva prevención, defensa, cuidado y ordenanza continúa siendo un objetivo no alcanzado (León y Cabrera, 2016). Por este motivo, es indispensable el ajuste a las instrucciones de captura de datos y cifras para que la información sea más real, útil, eficiente y se alinee correctamente a la violencia que se comete hacia las mujeres (García e Ibarra, 2017).

Según Menéndez, Pérez y Lorence (2013) los maltratos sufridos por las mujeres en poder de sus compañeros o excompañeros sentimentales son un fenómeno presentado de forma universal en cierta medida, donde no se tiene en cuenta clases sociales, ni un grupo específico de la población. Sin importar el género, los miembros de la pareja a veces poseen conductas violentas con un patrón de comportamiento de control o agresión física (Arnoso, Ibabe, y Elgorriaga, 2017).

Cuando se buscan datos de violencia entre pareja, contra la mujer específicamente con condición de gestante, no se consiguen cifras exactas que detallen causas, riesgos, patrones de conducta u otros aspectos de la violencia en mujeres en este estado (Ruiz, Gonzalez, Lopez, Iglesias, y Rubio, 2010). A raíz de la afirmación anterior, se demuestra la necesidad de un trabajo en equipo de observatorios y entidades delegadas, para que los cálculos sean más exactos, más detallados y se permita el acceso a consolidados de mayor confianza a escala nacional en cada uno de los Departamentos. Las políticas públicas se sustentan de los datos de los observatorios y estos son aún muy inseguros (Londoño, Giraldo, Montoya, y Ortega, 2014), motivo por el cual se ve la conveniencia de realizar estudios que permitan comprender los riesgos de violencia hacia la mujer en estado de embarazo. 
En algunos estudios cualitativos, se señala que los casos de violencia contra la mujer empiezan con pequeños detalles desde el noviazgo que a veces no son percibidos como violencia, motivo por el cual hay propuestas para evaluar y analizar los factores desde esta etapa. Por ejemplo, en la Universidad de Sevilla, España, propusieron el diseño y validación de un instrumento para detectar la violencia ejercida y padecida en las relaciones de parejas jóvenes, dónde se obtuvieron tres dimensiones principales: agresión física y sexual, conductas de control y abuso psicoemocional (García, Rodriguez, y Porcel, 2018). El mal trato hacia la mujer, suele iniciar con conductas de abuso emocional y psicológico, difíciles de detectar, ya que se esconden tras la apariencia de afecto. Estas conductas tóxicas de restricción y control van desmejorando la habilidad decisoria y autónoma de las mujeres, (Yugueros, 2014) y en una mujer embarazada causa muchísima más afectación no solo para ella sino también para su bebé. Se ha demostrado que la violencia hacia la pareja en la etapa de embarazo aumenta la probabilidad de aborto (Martínez, Aguilera, y López, 2014) y que la depresión y depresión posparto causa afectaciones emocionales en la gestante (Lafaurie, 2015). La literatura científica indica que existe variedad de estudios relacionados con la depresión postparto (Ramírez, 2012; Zapata, 2013; Ballester, 2014).

Estudios también demuestran que mujeres jóvenes que sufren violencia experimentan autoestima baja (Navarro, Rodríguez, y Hernández, 2017). De cara a prevenir y paliar los efectos de la violencia de pareja en adolescentes (Yago y Tomás, 2015), se proponen "programas de prevención que incluyan dentro de sus áreas de actuación acciones de educación emocional, que ayuden a mejorar el autoconcepto emocional de las víctimas" (Penado y Rodicio, 2017, p. 107). La principal ayuda que se les debe brindar a las mujeres víctimas de violencia de pareja es el apoyo social y psicológico; en estudios se demuestra que las mujeres que han buscado apoyo tienen menos probabilidad de ser violentadas que las que no buscan ayuda (Castaño, Ruíz, y Montero, 2008).

\section{Materiales y métodos}

Tipo de estudio: El presente estudio fue cuantitativo, de tipo descriptivo con corte transversal. Se definió, se clasificó y se caracterizó el objeto 
de estudio con el fin de establecer una medición o una cuantificación de la prevalencia a la exposición de riesgos y evaluar el impacto de violencia contra las mujeres gestantes y lactantes de Sincelejo por parte de su pareja.

Población: La población está conformada por las mujeres gestantes y lactantes del municipio de Sincelejo.

Muestra: La muestra estuvo conformada por 50 mujeres gestantes y lactantes del barrio Altos de la Sabana del Municipio de Sincelejo, Nivel 1, cuyas edades oscilan entre los 18 a 36 años, pertenecientes al programa de madres FAMI (familia, mujer e infancia) del Bienestar Familiar, dónde se les forma en autocuidado, estrategias y orientación del conflicto intrafamiliar (Fundación paz y bien, 2017).

Instrumentos: Se hizo uso de la escala de valoración de riesgo de violencia contra la pareja, más conocida como S.A.R.A.P (The Spousal Assault Risk Assessment) (Kropp y HArt, 2000). El instrumento consta de 20 ítem agrupados en cuatro sesiones que estiman el grado de historial delictivo del agresor, sus características psicosociales, su histórico de agresiones hacia la pareja y el delito o la agresión que incentiva la estimación. El Alfa de Crombach de la prueba fue de 0,89\%.

Tipo de análisis: El tipo de análisis utilizado corresponde a análisis estadísticos descriptivos donde se describen y resumen los datos obtenidos sobre riesgo de violencia contra la mujer gestante o lactante a través del programa de análisis estadístico SPSS. Adicional a la aplicación del instrumento, se recolectaron las cifras estadísticas proporcionadas por el Instituto Nacional de Medicina Legal y Ciencias Forenses en su informe preliminar para el año 2017, con el fin de realizar análisis estadístico y determinar características principales de las víctimas y los agresores de este tipo de violencia. Este estudio se utilizó, ya que es una de las fuentes más confiables de los observatorios; sus cifras son representación de todos los casos atendidos por este Instituto en el trascurrir del año 2017, la presentación del informe permite visualizar el contexto de la violencia contra la mujer desde diferentes ángulos ya que su presentación esta por variables haciéndolo útil y de fácil acceso. 


\section{Resultados}

1. A continuación, se presentan los resultados obtenidos con la aplicación de la escala de valoración de riesgo de violencia contra la pareja S.A.R.A.P:

Tabla 1. Historial de violencia

\begin{tabular}{|l|c|c|}
\hline & Frecuencia & Porcentaje \\
\hline Bajo & 22 & $44 \%$ \\
\hline Moderado & 18 & $36 \%$ \\
\hline Alto & 10 & $20 \%$ \\
\hline Total & 50 & $100 \%$ \\
\hline
\end{tabular}

Fuente: Elaboración propia.
Gráfico 1.

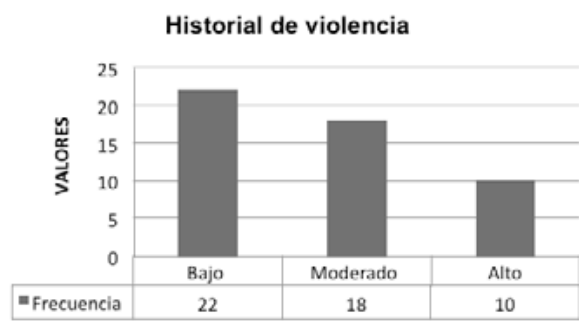

En la dimensión historial de violencia de la pareja se obtuvo un resultado de $44 \%$ para riesgo bajo, $36 \%$ para riesgo moderado y $20 \%$ para riesgo alto (Tabla 1 y Gráfico 1), indicando con ello que es mayor el porcentaje de mujeres que manifiesta que el agresor no ha tenido historial de violencia hacia su familia o hacia ellas mismas en calidad de víctimas.

Para el análisis del delito/agresión actual, (Tabla 2 y Gráfico 2) se detecta un riesgo elevado en este ítem con un 46\%, de riesgo alto, riesgo moderado del $36 \%$ y riesgo bajo del $18 \%$. Este resultado indica que es mayor el porcentaje de mujeres que manifiestan haber sido víctimas de violencia sexual, física o psicológica.

Tabla 2. Delito de agresión actual

\begin{tabular}{|l|c|c|}
\hline & Frecuencia & Porcentaje \\
\hline Bajo & 9 & $18 \%$ \\
\hline Moderado & 18 & $36 \%$ \\
\hline Alto & 23 & $46 \%$ \\
\hline Total & 50 & $100 \%$ \\
\hline
\end{tabular}

Fuente: Elaboración propia.
Gráfico 2.

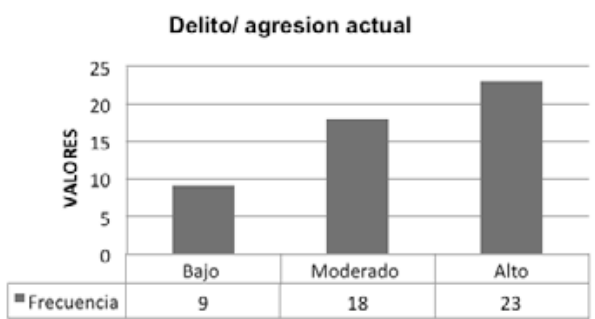


Para evaluar el historial delictivo, teniendo en cuenta las respuestas de las participantes, se detecta un riesgo bajo con un 44\%, 36\% de riesgo moderado y $20 \%$ de riesgo alto (Tabla 3 y Gráfico 3).

Tabla 3. Historial delictivo

\begin{tabular}{|l|c|c|}
\hline \multicolumn{1}{|c|}{ Nivel } & Frecuencia & Porcentaje \\
\hline Bajo & 22 & $44 \%$ \\
\hline Moderado & 18 & $36 \%$ \\
\hline Alto & 10 & $20 \%$ \\
\hline Total & 50 & $100 \%$ \\
\hline
\end{tabular}

Fuente: Elaboración propia.
Gráfico 3.

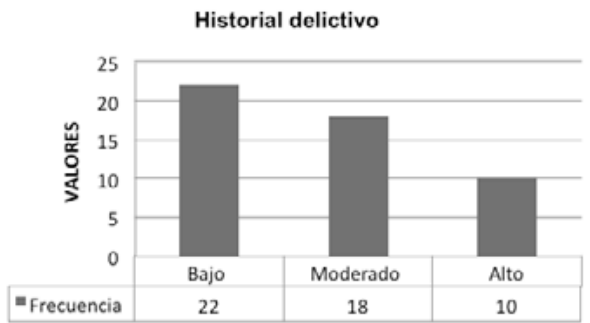

Por último, se evaluó el ajuste psicosocial (Tabla 4 y Gráfico 4), donde predomina el nivel alto con un $42 \%$, nivel medio con $36 \%$ y bajo con $22 \%$.

Tabla 4. Ajuste Psicosocial

Gráfico 4.

\begin{tabular}{|l|c|c|}
\hline \multicolumn{1}{|c|}{ Nivel } & Frecuencia & Porcentaje \\
\hline Bajo & 11 & $22 \%$ \\
\hline Moderado & 18 & $36 \%$ \\
\hline Alto & 21 & $42 \%$ \\
\hline Total & 50 & $100 \%$ \\
\hline
\end{tabular}

Fuente: Elaboración propia.

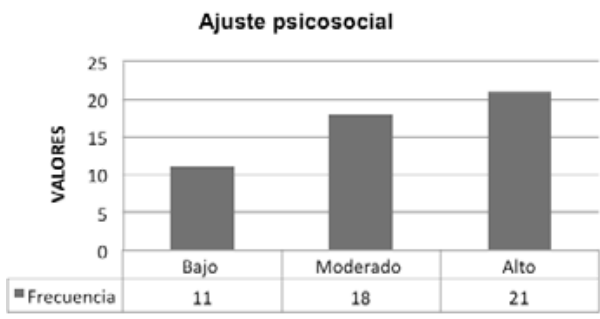

2. A continuación, se presentan los resultados del análisis estadístico de las cifras presentadas por el Instituto Nacional de Medicina Legal y Ciencias Forenses (INMLCF) tomando como base el informe preliminar de lesiones no fatales a causa de violencia en pareja a nivel Nacional en Colombia de enero a diciembre de 2017 (INMLCF, 2017).

Según la Tabla 5, donde se muestran los valores obtenidos entre 42.492 mujeres que acudieron a medicina legal por violencia de pareja, el 3\% era menor de edad en comparación al 97 \% que corresponde a población de mujeres mayores de edad. Según la Tabla 6, el mayor porcentaje de población se encuentra en el rango de edad de 25 a 29 años seguido por los 20 a los 24 y, de 30 a 34 es donde hay más riesgo de sufrir violencia al interior de la pareja. 
Tabla 5. Grupo mayor / menor de edad

\begin{tabular}{|l|c|c|}
\hline \multicolumn{3}{|c|}{ Variable grupo mayor/menor de edad } \\
\hline Violencia de pareja VIF & $\mathbf{4 2 . 5 9 2}$ & $\%$ \\
\hline Menores de edad ( <18 años) & 1.377 & $3 \%$ \\
\hline Mayores de edad ( >18 años) & 41.215 & $97 \%$ \\
\hline
\end{tabular}

Fuente: Tomado de informe preliminar de lesiones no fatales a causa de violencia en pareja de Enero a Diciembre de 2017.

Tabla 6. Variable grupo edad

\begin{tabular}{|c|c|c|}
\hline \multicolumn{3}{|c|}{ VARIABLE: GRUPO DE EDAD } \\
\hline 4 VIOLENCIA DE PAREJA (VIF) & 42.592 & $\%$ \\
\hline (10 a 14$)$ & 84 & $0,20 \%$ \\
\hline (15 a 17) & 1.293 & $3,04 \%$ \\
\hline (18 a 19) & 2.319 & $5,44 \%$ \\
\hline$(20$ a 24$)$ & 9.323 & $21,89 \%$ \\
\hline (25 a 29) & 9.407 & $22,09 \%$ \\
\hline (30 a 34$)$ & 7.634 & $17,92 \%$ \\
\hline (35 a 39) & 5.442 & $12,78 \%$ \\
\hline$(40$ a 44$)$ & 3.105 & $7,29 \%$ \\
\hline (45 a 49) & 1.944 & $4,56 \%$ \\
\hline (50 a 54) & 1.152 & $2,70 \%$ \\
\hline (55 a 59$)$ & 478 & $1,12 \%$ \\
\hline$(60$ a 64$)$ & 233 & $0,55 \%$ \\
\hline (65 a 69) & 96 & $0,23 \%$ \\
\hline (70 a 74$)$ & 56 & $0,13 \%$ \\
\hline (75 a 79$)$ & 18 & $0,04 \%$ \\
\hline (80 y más) & 8 & $0,02 \%$ \\
\hline
\end{tabular}

Fuente: Tomado de informe preliminar de lesiones no fatales a causa de violencia en pareja de Enero a Diciembre de 2017.

Tomando como base la escolaridad, a través de la Tabla 7 se puede deducir que las mujeres que están más propensas al riesgo de ser víctimas de violencia en la pareja se encuentran en nivel escolar de básica secundaria con un 52,85\%, seguido de las mujeres que solo tienen primaria con el 
29,38\%. Según la Tabla 8, respecto al estado conyugal, la violencia es más frecuente en la unión libre con un 53,23\% seguido del estado soltera, con el $37,45 \%$.

En cuanto al estado de la pareja que es reportado más frecuentemente, el 65,3\% corresponde al compañero permanente mientras que el $40.0 \%$ al excompañero permanente (Tabla 9).

Tabla 7. Variable escolaridad

\begin{tabular}{|c|c|c|}
\hline \multicolumn{3}{|l|}{ VARIABLE: ESCOLARIDAD } \\
\hline 4 VIOLENCIA DE PAREJA (VIF) & 49.423 & $\%$ \\
\hline Educación inicial y educación preescolar & 2.522 & $5,92 \%$ \\
\hline Educación básica primaria & 12.512 & $29,38 \%$ \\
\hline Educación básica secundaria o secundaria baja & 22.510 & $52,85 \%$ \\
\hline Educación media o secundaria alta & 2.330 & $5,47 \%$ \\
\hline Educación técnica profesional y tecnológica & 8.431 & $19,79 \%$ \\
\hline Universitario & 237 & $0,56 \%$ \\
\hline Especialización, Maestría o equivalente & 91 & $0,21 \%$ \\
\hline Doctorado o equivalente & 19 & $0,04 \%$ \\
\hline Sin escolaridad & 363 & $0,85 \%$ \\
\hline Sin información & 408 & $0,96 \%$ \\
\hline
\end{tabular}

Fuente: Tomado de informe preliminar de lesiones no fatales a causa de violencia en pareja de Enero a Diciembre de 2017.

Tabla 8. Variable estado conyugal

\begin{tabular}{|l|c|c|}
\multicolumn{3}{c}{ VARIABLE: ESTADO CONYUGAL } \\
\hline 4 VIOLENCIA DE PAREJA (VIF) & $\mathbf{4 9 . 4 2 3}$ & $\mathbf{\%}$ \\
\hline Soltero (a) & 15.950 & $37,45 \%$ \\
\hline Unión libre & 22.673 & $53,23 \%$ \\
\hline Casado (a) & 6.222 & $14,61 \%$ \\
\hline Separado (a), Divorciado (a) & 4.296 & $10,09 \%$ \\
\hline Viudo (a) & 109 & $0,26 \%$ \\
\hline Sin información & 173 & $0,41 \%$ \\
\hline
\end{tabular}

Fuente: Tomado de informe preliminar de lesiones no fatales a causa de violencia en pareja de Enero a Diciembre de 2017. 
Tabla 9. Tipo de pareja

\begin{tabular}{|l|c|}
\hline \multicolumn{1}{|c|}{ 4 VIOLENCIA DE PAREJA } & $\mathbf{3 7 7}$ \\
\hline Pareja o ex pareja & 377 \\
\hline Amante & 180 \\
\hline Compañero (a) permanente & 1 \\
\hline Ex-amante & 179 \\
\hline Ex-compañero (a) permanente & 10 \\
\hline Ex- novio (a) & 7 \\
\hline Novio (a) & \\
\hline Sin información & \\
\hline $\begin{array}{l}\text { Fuente: Tomado de informe preliminar de lesiones no fatales } \\
\text { a causa de violencia en pareja de Enero a Diciembre de } 2017 .\end{array}$
\end{tabular}

3. A continuación, se presentan los resultados del análisis estadístico de las cifras presentadas por el Instituto Nacional de Medicina Legal y Ciencias Forenses (INMLCF) tomando como base el informe preliminar de lesiones no fatales a causa de violencia en pareja de enero a diciembre de 2017, donde se mide solo la participación de Sincelejo dentro del $100 \%$ de informes reportados en el Departamento de Sucre (INMLCF, 2017).

Según la Tabla 10, en rango de edades, el 61\% del 100\% de los casos de violencia contra la pareja en la mujer reportados en el Departamento de Sucre, corresponden al municipio de Sincelejo, de los cuales el 3\% de los casos son en menores de edad y el $58 \%$ en personas mayores de edad. La gran mayoría están ubicados en el rango de edades de 20 a 34 años (Tabla 11) y el rango de estudio de estas mujeres en su mayor proporción es de básica secundaria y primaria (Tabla 12). En tal sentido, haciendo el comparativo con la estadística arrojada a nivel nacional, se evidencia que el comportamiento de estas características para la población de Sincelejo es igual.

El estado conyugal en la ciudad de Sincelejo (Tabla 13) indica que las mujeres que más riesgo corren de presentar violencia de pareja son las mujeres en Unión libre y solteras. 
Tabla 10. Variable grupo mayor/menor

\begin{tabular}{|l|c|c|c|}
\hline \multicolumn{5}{|c|}{ VARIABLE: GRUPO MAYOR/MENOR DE EDAD } \\
\hline 4 VIOLENCIA DE PAREJA (VIF) & $\mathbf{3 7 7}$ & $\mathbf{6 1 4}$ & $\mathbf{6 1}$ \\
\hline a) Menores de Edad (<18 años) & 19 & 29 & 3 \\
\hline b) Mayores de Edad (>18 años) & 358 & 585 & 58 \\
\hline
\end{tabular}

Fuente: Tomado de informe preliminar de lesiones no fatales a causa de violencia en pareja de Enero a Diciembre de 2017.

Tabla 11. Variable grupo de edad

\begin{tabular}{|l|c|c|c|}
\hline \multicolumn{4}{|c|}{ VARIABLE GRUPO DE EDAD } \\
\hline 4 VIOLENCIA DE PAREJA (VIF) & $\mathbf{3 7 7}$ & $\mathbf{6 1 4}$ & $\mathbf{6 1 \%}$ \\
\hline (10 a 14) & 1 & 1 & 0 \\
\hline (15 a 17) & 18 & 28 & 18 \\
\hline (18 a 19) & 15 & 29 & 0,54 \\
\hline (20 a 24) & 76 & 135 & 2,62 \\
\hline (25 a 29) & 87 & 135 & 0,64 \\
\hline (30 a 34) & 79 & 119 & 0,59 \\
\hline (35 a 39) & 45 & 72 & 0,38 \\
\hline (40 a 44) & 22 & 43 & 0,31 \\
\hline (45 a 49) & 16 & 25 & 0,37 \\
\hline (55 a 59) & 3 & 4 & 0,2 \\
\hline (60 a 64) & 3 & 6 & 0,75 \\
\hline (65 a 69) & 1 & 1 & 0,17 \\
\hline (70 a 74) & 1 & 1 & 1 \\
\hline (75 a 79) & - & - & 0 \\
\hline (80 y más) & - & - & 0 \\
\hline
\end{tabular}

Fuente: Tomado de informe preliminar de lesiones no fatales a causa de violencia en pareja de Enero a Diciembre de 2017. 
Tabla 12. Variable escolaridad

\begin{tabular}{|l|c|c|c|}
\hline 4 VIOLENCIA DE PAREJA (VIF) & $\mathbf{3 7 7}$ & $\mathbf{6 1 4}$ & $\mathbf{6 1 \%}$ \\
\hline Educación inicial y educación preescolar & 20 & 44 & 0,03 \\
\hline $\begin{array}{l}\text { Educación básica primaria } \\
\text { Educación básica secundaria o secundaria }\end{array}$ & 97 & 167 & 0,16 \\
\hline $\begin{array}{l}\text { baja } \\
\text { Educación media o secundaria alta }\end{array}$ & 6 & 349 & 0,36 \\
\hline $\begin{array}{l}\text { Educación técnica profesional y tecnológica } \\
\text { Universitario }\end{array}$ & 27 & 33 & 0,01 \\
\hline Especialización, Maestría o equivalente & 2 & 3 & 0 \\
\hline Doctorado o equivalente & & 2 & 0 \\
\hline Sin escolaridad & 2 & 7 & 0 \\
\hline Sin información & & & 0 \\
\hline
\end{tabular}

Fuente: Tomado de informe preliminar de lesiones no fatales a causa de violencia en pareja de Enero a Diciembre de 2017.

Tabla 13. Variable estado conyugal

\begin{tabular}{|l|c|}
\hline \multicolumn{2}{|c|}{ VARIABLE: ESTADO CONYUGAL } \\
\hline 4 VIOLENCIA DE PAREJA (VIF) & $\mathbf{3 7 7}$ \\
\hline Soltero (a) & 108 \\
\hline Unión libre & 191 \\
\hline Casado (a) & 33 \\
\hline Separado (a), Divorciado (a) & 42 \\
\hline Viudo (a) & 3 \\
\hline Sin información & \\
\hline
\end{tabular}

Fuente: Tomado de informe preliminar de lesiones no fatales a causa de violencia en pareja de Enero a Diciembre de 2017.

Al igual que la estadística nacional para Sincelejo la persona que más perpetua el acto de violencia es el compañero permanente o el excompañero de la mujer (Tabla 14). 
Tabla 14. Tipo de pareja

\begin{tabular}{|l|c|}
\hline 4 VIOLENCIA DE PAREJA & $\mathbf{3 7 7}$ \\
\hline Pareja o ex pareja & $\mathbf{3 7 7}$ \\
\hline Amante & \\
\hline Compañero (a) permanente & 180 \\
\hline Ex-amante & 1 \\
\hline Ex-compañero (a) permanente & 179 \\
\hline Ex- novio (a) & 10 \\
\hline Novio (a) & 7 \\
\hline Sin información & \\
\hline
\end{tabular}

Fuente: Tomado de informe preliminar de lesiones no fatales a causa de violencia en pareja de Enero a Diciembre de 2017.

\section{Discusión}

1. La visión de la violencia en la pareja contra la mujer en la actualidad

En la convivencia en pareja es muy probable la ocurrencia de disgustos por diferencias presentadas entre los miembros (Muñoz y Echeburua, 2016). La violencia de pareja donde las mujeres son las afectadas, constituye un problema social grave que inquieta a un grupo importante de individuos; se trata de un acontecimiento muy diverso y universal sobre todo si se habla de las características de las víctimas (Menendez, Pérez y Lorence, 2013). “La violencia contra la mujer está presente en la mayoría de las sociedades pero a menudo no es reconocida y se acepta como parte del orden establecido" (OMS, 1998, p. 2). Hasta hace pocos años se empezó a ver la violencia contra la mujer como un tema de alto impacto, es por esto que aún sigue prevaleciendo los mitos y el machismo en la sociedad que en ocasiones admite que esta situación se siga presentando. La sociedad, diferentes entidades y el estado, realizan esfuerzos mancomunados encaminados a disminuir este suceso, colocando a disposición herramientas de prevención, mecanismos de denuncia, intervención policial, así como la elaboración de leyes y políticas públicas específicas en la materia. Lo anterior con consecuencias discutibles respecto al control del riesgo experimentado (Contreras, 2014). 
Aunque existen leyes que protegen a la mujer, las mujeres aún viven con miedo de denunciar. A las víctimas les cuesta más abandonar o denunciar al agresor, por el miedo a las agresiones físicas que les infunden y por las técnicas de manipulación utilizadas. (Boira, Carbajosa, y Méndez, 2016).

\section{Causas de la existencia de la violencia en la pareja contra la mujer}

En la actualidad sigue el debate sobre las causas y consecuencias de la existencia de la violencia contra la mujer en la pareja; el debate, por ejemplo, sobre el motivo o raíz de origen cultural o congénito de la violencia sigue arraigado en nuestra sociedad y demuestra la diversidad de causas y consecuencias que en la literatura científica se han abordado, relacionado y establecido con la presencia de conductas violentas (biológicas, psicológicas o sociales) (Alonso y Castellanos, 2006; Domínguez, Martínez, Vásquez, y Rodríguez, 2017).

Este comportamiento de violencia se debe también a los falsos conceptos que aún tienen los hombres de la sociedad de considerar a las mujeres como un objeto suyo del cual disponen a su antojo (Cabrera, Alonso, y Mederos, 2012). El bajo nivel educativo y una permanencia mayor en casa desempeñándose como amas de casa es uno de los factores de riesgo más determinantes de la presencia de violencia en la pareja, la pobreza es causal de la existencia o generación de desigualdad entre ambos sexos, donde se favorece la aparición de violencia de genero donde la víctima es la mujer (SciLogs, 2013).

"El nivel socio-comunitario, el bajo nivel educativo, el bajo desarrollo económico del país, pocos derechos sociales, la falta de democratización del Estado, la cultura del honor y las culturas masculinas — con actitudes sexistas y favorables hacia la violencia en el país- son factores de riesgo" (Echeburúa, Puente, Ubillos y Páez, 2016, p. 295). Según otros estudios, otros factores que se categorizan como asociados son la violencia durante la infancia el consumo frecuente de alcohol del hombre y capacidad limitada de toma de decisiones (Jaen, Rivera, Amorin y Rivera, 2015), nivel socioeconómico bajo, antecedentes de violencia (Sandoval, Jiménez, Rovira y Hernández, 2017). 
La situación es de bastante trascendencia, teniendo en cuenta que "cada 12 minutos, por lo menos una mujer es violentada por su pareja o expareja” (Corporación Sisma Mujer, 2017, p. 5).

\section{Consecuencias de la violencia en la pareja contra la mujer}

La violencia contra la mujer es expresada por medio de actos y palabras que ocasionan que esta se sienta violentada psicológicamente, humillada, ignorada y depreciada con las actitudes de su pareja, evidenciándose sentimientos de ira, rabia, depresión y angustia por el trato que recibe. Los hallazgos de esta investigación en lo relacionado con el alto riesgo de violencia física, sexual o Psicológica en la dimensión delito/agresión y ajuste psicosocial, son coherentes con otras investigaciones realizadas en Colombia, citando específicamente el tema de la mujer gestante, "en una investigación realizada en Bogotá, Colombia, se buscó establecer la relación entre la violencia de pareja contra la mujer y la aparición de depresión postparto en 81 mujeres puérperas; se logró establecer que el 54\% de las participantes fue maltratada, $17 \%$ presentó abuso físico por su pareja, 54\% presentó abuso no físico y el 33\% de las participantes de la investigación presentó sintomatología depresiva en el postparto" (Ramírez, 2012, p. 9).

Se determina que el historial de violencia e historial delictivo en Sincelejo está en la escala de calificación bajo en comparación a lo detallado en los estudios nombrados al interior del presente artículo, donde se indica que los índices de violencia contra la mujer han incrementado año tras año; puede que estos índices siempre hayan sido elevados pero desde hace unos pocos años se esté haciendo público este acto como problema social. En el caso de estudio se da como resultado en la valoración de delito y /o agresión actual y ajuste psicosocial un nivel alto, esto soporta la afirmación anterior dando a conocer los datos que hoy en día se están presentando.

Según el Departamento de Justicia de Cataluña en España para el año 2006 se indica que la frecuencia de la violencia hacia la mujer incrementa cuando ha existido una relación abusiva, muchas veces son actos o sucesos permitidos y no denunciados por la mujer que van aumentando con el transcurrir del tiempo. 
Los datos arrojados en el caso de estudio son bastante bajos en comparación en las cifras informadas en el informe preliminar del INMLCF. Cada vez se hace más evidente la creciente necesidad de diseñar e implementar formas más eficientes de prevención y reacción de la violencia contra la mujer.

Como se ha venido estudiando, las variables y factores que actúan en la violencia contra la mujer son diversas y por más estudios que se han realizado incluyendo el caso de estudio propuesto, no se pueden predecir las conductas violentas, solo se puede valorar probabilidades de riesgo de ocurrencia, es decir; estimar riesgos relativos.

La violencia sufrida por las mujeres tiene importantes consecuencias en la salud y supone un patrón de comportamiento que se expresa, entre otros, a través de la satisfacción en la pareja o el tipo de amor en la relación (Diaz, Estévez, Moñene, y Linares, 2018). En estudios como los de Limiñana, Martínez y Mateo (2018) y Esparza y Aroca (2017) se resalta que los comportamientos de los niños que se encuentran en contextos familiares donde se presencia violencia de género también tienen repercusiones en su conducta infantil y competencias parentales (Valdés, Martínez, y Carlos, 2018).

La violencia ha pasado de este maltrato psicológico al maltrato físico donde en los peores escenarios, se ha convertido en feminicidio entendiendo el término como "un tipo de homicidio específico en el que un varón, asesina a una mujer o niña por ser de sexo femenino. A diferencia de otros tipos de asesinato, los feminicidios suelen ocurrir en el hogar como consecuencia de la violencia de género. También se categorizan dentro de los crímenes de odio, dado que se dan en un contexto en el que lo femenino ha sido estigmatizado durante años" (Figueroba, 2015, p. 1). Estudios indican que la muerte de femeninas a manos de su pareja o expareja se incrementaron de manera gradual y alarmante (Reguera, Calvario, y Mora, 2014).

La Organización Mundial de la Salud (OMS) señala que, en 11 de 15 países examinados, el porcentaje de mujeres que alguna vez habían estado embarazadas y habían sido víctimas de violencia física durante al menos un embarazo superaba el 5\% (Moreto, 2013). 


\section{Conclusiones}

Los resultados obtenidos, se deben tomar como base de información sobre las características y circunstancias de la violencia en pareja hacia la mujer gestante y/o lactante. Es primordial para adaptar, teniendo en cuenta lo observado y los resultados obtenidos, las posibles medidas de prevención adecuadas y el tratamiento efectivo a esta problemática.

A pesar de la escasa información que se encuentra sobre estudios efectuados sobre el riesgo de violencia contra la pareja en mujeres gestantes y/o lactantes, los resultados obtenidos en el estudio de caso llevan a concluir que en la ciudad de Sincelejo a pesar de que la pareja presenta un grado bastante bajo tanto en riesgo por historial de violencia o riesgo actual, no se garantiza que durante el embarazo o después de él, en la etapa de lactancia se siga manteniendo ese mismo nivel, dado que los sujetos estudiados si presentan índices elevados de delincuencia. Las causas son hasta el momento desconocidas ya que no se han realizado estudios sobre los principales factores asociados a la violencia de la mujer durante el embarazo, pero si se asocian con la violencia contra la mujer a nivel nacional en Colombia o conlos datos obtenidos delas estadísticas de acuerdoalinforme del INMLCF los riesgos siguen siendo en edades de 20 a 34 años con estudios entre primaria y bachillerato, amas de casa en unión libre o solteras, que no generan ingresos donde sus principales victimarios son sus compañeros o excompañeros permanentes.

Esta investigación invita a los observatorios y entes encargados de velar por el bienestar de las mujeres embarazadas a investigar y a documentar más sobre la violencia en esta condición de gestación y/o lactancia para poder proponer detectar con mayor eficiencia los indicadores de probabilidad de ocurrencia, donde se cuente con validación externa y comparación de precisión diagnóstica (García, y otros, 2011). Es necesario actuar ante esta situación ya que no se afecta una sola vida.

Se recomienda brindar mayor apoyo y protección a las mujeres que son víctimas de tratos indebidos, degradantes e inhumanos caracterizando el tipo de violencia en las mujeres gestantes. Para que las mujeres en esta condición sientan respaldo y cumplimiento de los derechos humanos que 
les están siendo violados por parte de sus parejas (Organización Mundial Contra la Tortura, 2017) de igual forma no solo se aconseja brindar recuperación integral a la mujer agredida, también se propone brindar rehabilitación al agresor (Cruz, 2010). 


\section{Referencias bibliográficas}

Alonso, J., y Castellanos, J. (2006). Por un enfoque integral de la violencia familiar. Psychosocial Intervention, 15(3). Obtenido de http://scielo.isciii. es/scielo.php?script=sci_Arttextypid=S1132-05592006000300002

Arnoso, A., Ibabe, I., y Elgorriaga, E. (2017). El sexismo como predictor de la violencia de pareja en un contexto multicultural. Anuario de Psicología Jurídica, 9-20. doi:https://doi.org/10.1016/j.apj.2017.02.001

Ballester, A. (2014). Valoración psicológica en delitos de violencia de género mediante el Inventario Clínico y Multiaxial de Millon III (MCMIIII). Anuario de Psicología Jurídica, 9-18. doi:https://doi.org/10.1016/j. apj.2014.06.001

Boira, S., Carbajosa, P., y Méndez, R. (2016). Miedo, conformidad y silencio. La violencia en las relaciones de pareja en áreas rurales de Ecuador. Psychosocial Intervention, 9-17. doi:https://doi.org/10.1016/j. psi.2015.07.008

Cabrera, M., Alonso, H., y Mederos, M. (2012). Violencia contra la mujer en la comunidad. Medisan, 1-7. Obtenido de http://bvs.sld.cu/revistas/ san/vol_16_8_12/san12812.pdf

Castaño, J., Ruiz, I., y Montero, M. (2008). Apoyo social como factor protector frente a la violencia contra la mujer en la pareja. Gaceta Sanitaria, 527-533. doi:https://doi.org/10.1016/S0213-9111(08)75350-0

Cirici , R., Querol, N., y Ripoll, A. (2010). La consulta sanitaria: ¿un espacio privilegiado para la detección y el abordaje de la violencia de género? FMC -Formación Médica Continuada en Atención Primaria, 550-559. doi:https://doi.org/10.1016/S1134-2072(10)70213-9

Contreras, L. (2014). Factores de riesgo de homicidio de la mujer en la relación de pareja. Universitas Psychologica, 13(2), 681-692. doi:doi:10.11144/Javeriana.UPSY13-2.frhm

Corporación Sisma Mujer. (2017). La erradicación de las violencias contra las mujeres: una tarea inaplazable en la construcción de paz. Bogotá, 
D.C.: Sisma Mujer. Obtenido de http://www.sismamujer.org/25-denoviembre-de-2017-sisma-mujer/

Cruz, C. (2010). Perspectiva judicial de la violencia de pareja y doméstica en España. Revista Española de Medicina Legal, 93-97. doi:https://doi. org/10.1016/S0377-4732(10)70036-5

Diaz, M., Estévez, A., Moñene, J., y Linares, L. (2018). Las actitudes amorosas y la satisfacción en la pareja como factores intervinientes en la relación entre la violencia y las consecuencias en la salud de las mujeres. Ansiedad y Estrés. doi:https://doi.org/10.1016/j.anyes.2018.01.001

Domínguez , M., Martínez, I., Vásquez, F., y Rodríguez, M. (2017). Características y consecuencias de la violencia de género: estudio de casos confirmados por sentencia judicial. 115-122. doi:https://doi. org/10.1016/j.reml.2017.05.001

Echeburúa, E., Puente, A., Ubillos, S., y Páez, D. (2016). Factores de riesgo asociados a la violencia sufrida por la mujer en la pareja: Una revisión de meta-análisis y estudios recientes. Canales de psicologia, 32(1), 295-306. doi:http://dx.doi.org/10.6018/analesps.32.1.189161

Latorre-Iglesias, E. (2015). Anotaciones críticas sobre las cegueras educativas: Los desafíos de una educación de calidad en la era conceptual. Vis Iuris, Vol. 2 (4), p. 21.

Latorre-Iglesias, E.; Latorre-Iglesias, A. (2015). Re-educar la humanidad: La necesidad de emocionalidad en los profesores del derecho. Vis Iuris, Vol. 2 (3), p. 81.

Esparza, C., y Aroca, C. (2017). Menores que maltratan a sus progenitores: definición integral y su ciclo de violencia. Anuario de Psicología Jurídica. doi:https://doi.org/10.1016/j.apj.2017.01.001

Figueroba, A. (2015). Feminicidio (asesinatos a mujeres): definición, tipos y causas. PSICOLOGÍA FORENSE Y CRIMINALÍSTICA. Obtenido de https://psicologiaymente.net/forense/feminicidio 
Fundación paz y bien. (2017). Fundación paz y bien. Obtenido de Fundación paz y bien: http://fundacionpazybien.org/madres-fami/

Garcia, L., Torres, A., Navarro, P., Ascaso, C., Imaz, M., y Herreras, Z. (2011). Validación y comparación de cuatro instrumentos para la detección de la violencia de pareja en el ámbito sanitario. Medicina Clínica, 390-397. doi:https://doi.org/10.1016/j.medcli.2010.11.038

Garcia, M., y Ibarra, M. (2017). Detrás de las cifras de violencia contra las mujeres en Colombia. Sociedad y Economia, 41-64. Obtenido de http:// www.scielo.org.co/pdf/soec/n32/1657-6357-soec-32-00041.pdf

García, M., Rodríguez, J., y Porcel, A. (2018). Diseño y validación de la escala para la detección de violencia en el noviazgo en jóvenes en la Universidad de Sevilla. Gaceta Sanitaria, 1. doi:https://doi.org/10.1016/j. gaceta.2017.09.006

Hodson. (2010). Uplap. Recuperado el 01 de Agosto de 2016, de http:// catarina.udlap.mx/u_dl_a/tales/documentos/lii/gomez_g_jm/ capitulo3.pdf

INMLCF. (2017). www.medicinalegal.gov.co. Obtenido de http://www. medicinalegal.gov.co/observatorio-de-violencia

Jaen, C., Rivera, S., Amorin, E., y Rivera, L. (2015). Violencia de Pareja en Mujeres: Prevalencia y Factores Asociados. Acta de Investigación Psicológica, 2224-2239. doi:https://doi.org/10.1016/S2007-4719(16)30012-6

Kropp, R., y HArt, S. (2000). The Spousal Assault Risk Assessment (SARA) Guide: Reliability and Validity in Adult Male Offenders. Law and Human Behavior, 582. Obtenido de https://link.springer.com/ Article/10.1023/A:1005430904495

Lafaurie, M. (2015). Violencia de la pareja íntima durante el embarazo y sus repercusiones en la salud mental. Ciencia y Cuidado, 12(2), 100-116. doi:DOI: http://dx.doi.org/10.22463/17949831.512

León , A., y Cabrera, L. (2016). Ley 1257 Ocho años de Obstáculos en la protección integral para las mujeres victimas de violencias. Bogotá: Ediciones 
Antropos Ltda. Recuperado el 24 de febrero de 2018, de http://www. sismamujer.org/wp-content/uploads/2016/11/Ley-1257-ocho-a\%C3\%B1os-de-obstaculos-en-la-protecci\%C3\%B3n-integral-de-las-mujeres-v\%C3\%ADvtimas-de-violencias.pdf

Ley No 1257. (2008). Recuperado el 24 de 02 de 2018, de https://www. oas.org/dil/esp/LEY_1257_DE_2008_Colombia.pdf

Limiñana, A., Martínez, R., y Mateo, M. (2018). Problemas de conducta infantil y competencias parentales en madres en contextos de violencia de género. Gaceta Sanitaria, 35-40. doi:https://doi.org/10.1016/j.gaceta.2017.02.004

Londoño, B., Giraldo, S., Montoya, A., y Ortega, V. (2014). Violencia contra las mujeres en tres ciudades de Colombia: Pasto Partícipesgena y Cali. 2005-2009. Opinión Jurídica-Universidad de Medellin, 13(25), 35-50. Recuperado el 26 de 02 de 2018, de http://revistas.udem.edu. co/index.php/opinion/Article/view/860/828

Martínez, M., Aguilera, E., y López, C. (2014). Aborto y salud de la mujer. Gaceta Sanitaria, 496-497. doi:https://doi.org/10.1016/j.gaceta.2014.06.003

Menéndez, S., Pérez, J., y Lorence, B. (2013). La violencia de pareja contra la mujer en España: Cuantificación y caracterización del problema, las víctimas, los agresores y el contexto social y profesional. ELSEVIER, 41-53. doi:DOI: http://dx.doi.org/10.5093/in2013a6

Menendez, S., Pérez, J., y Lorence, L. (2013). La violencia de pareja contra la mujer en España: Cuantificación y caracterización del problema, las víctimas, los agresores y el contexto social y profesional. Psychosocial Intervention, 41-53. doi:https://doi.org/10.5093/in2013a6

Moreto, A. (01 de septiembre de 2013). Desmontando mitos: La violencia de genero durante el embarazo. Obtenido de Universidad de Salamanca: https://gredos.usal.es/jspui/bitstream/10366/122420/1/TFM_ IzquierdoMoreta_Desmontando.pdf 
Muñoz , J., y Echeburua, E. (2016). Diferentes modalidades de violencia en la relación de pareja: implicaciones para la evaluación psicológica forense en el contexto legal español. 2-12. doi:https://doi.org/10.1016/j. apj.2015.10.001

Navarro, N., Rodríguez, O., y Hernández, F. (2017). Autoestima, violencia de pareja y conducta sexual en mujeres indígenas. Enfermeria Universitaria, 162-169. doi:https://doi.org/10.1016/j.reu.2017.05.002

OMS. (1998). Violencia contra la mujer, un tema prioritario. Planetaria. Obtenido de http://www.who.int/gender/violence/violencia_infopack1.pdf

Organización Mundial Contra la Tortura. (2017). Red Sos Tortura. Obtenido de http://www.omct.org/es/violence-against-women/

Penado, M., y Rodicio, L. (2017). Análisis del autoconcepto en las víctimas de violencia de género entre adolescentes. Suma Psicológica, 107-114. doi:https://doi.org/10.1016/j.sumpsi.2017.08.001

Ramirez, E. (2012). Relación entre violencia de pareja contra la mujer y a aparición de la depresión postparto. Bogotá, Colombia. Recuperado el 26 de 02 de 2018, de http://www.bdigital.unal.edu. co/8905/1/539374.2012.pdf

Reguera, M., Calvario, J., y Mora, F. (2014). Violencia contra las mujeres en el noreste de México: elementos para su contextualización. Acta Sociológica, 65, 65-95. doi:https://doi.org/10.1016/S0186-6028(14)70237-3

Ruiz, M., González, P., López, J., Iglesias, M., y Rubio, C. (2010). Violencia de género en un área de salud. SEMERGEN - Medicina de Familia, 202-207. doi:https://doi.org/10.1016/j.semerg.2010.02.004

Saenz, B., Heras, J., Otero, L., y Vives, C. (2016). Perfil sociodemográfico del feminicidio en España y su relación con las denuncias por violencia de pareja. Gaceta Sanitaria, 272-278. doi:https://doi.org/10.1016/j.gaceta.2016.03.004

Sandoval, L., Jiménez, M., Rovira, G., y Hernández, O. (2017). Violencia de pareja: tipo y riesgos en usuarias de atención primaria de salud en Cancún, Quintana Roo, México. Atención Primaria, 465-472. doi:https://doi.org/10.1016/j.aprim.2016.09.013 
Sarduy, Y. (2007). El análisis de información y las investigaciones cuantitativa y cualitativa. Cubana salud pública, 33. Obtenido de http:// bvs.sld.cu/revistas/spu/vol33_3_07/spu20207.htm

SciLogs. (2013). De la violencia contra las mujeres. Investigación y ciencia, 1. Obtenido de https://www.investigacionyciencia.es/blogs/psicologia$\mathrm{y}$-neurociencia/30/posts/de-la-violencia-contra-las-mujeres-11663

Valdés, A., Martínez, B., y Carlos, E. (2018). El rol de las prácticas docentes en la prevención de la violencia escolar entre pares. Revista de Psicodidáctica, 33-38. doi:https://doi.org/10.1016/j.psicod.2017.05.006

Yago, T., y Tomás, C. (2015). Violencia de pareja hacia las adolescentes y jóvenes atendidas en una consulta de anticoncepción e indicadores de riesgo. Revista Española de Medicina Legal, 41(3), 103-110. doi:https:// doi.org/10.1016/j.reml.2015.02.003

Yugueros, A. (2014). La violencia contra las mujeres: conceptos y causas. Revista Castellano- Manchega de ciencias sociales(18), 147-159. Obtenido de http://www.redalyc.org/html/3221/322132553010/

Zapata, F. (2013). Violencia de pareja en el Departamento del Quindío, Colombia. Salud Pública, 15(2), 247-257. Obtenido de https://revistas. unal.edu.co/index.php/revsaludpublica/Article/view/40848/62087 